\section{Synthese von positionsmarkierter Saccharose aus freier Glucose}

\section{Von Heinz Kauss}

Institut für Angewandte Botanik, Technische Hochschule München

(Z. Naturforschg. 17 b, 698-699 [1962] ; eingegangen am 1. August 1962)

Für stoffwechselphysiologische Untersuchungen benötigten wir Saccharose, die nur im Kohlenstoffatom 1 des Glucoseteils mit ${ }^{14} \mathrm{C}$ markiert ist. Bisher benützte man zur Synthese von Saccharose, die das radioaktive Isotop nur im Glucose- oder Fructoseteil enthält, das Ferment Saccharose-Phosphorylase aus Pseudomonas ${ }^{1}$. Zur Markierung des Glucoseteils wurde dabei stets nur undefiniert markiertes Glucose-1- $\mathrm{PO}_{4}$ aus Photosyntheseprodukten verwendet. Die Abwandlung dieses Verfahrens für unsere Zwecke hätte die Herstellung von Glucose-1- $\mathrm{PO}_{4}$ aus käuflicher positionsmarkierter Glucose vorausgesetzt. Mit isolierten Enzymen ist dies sehr umständlich und schwierig. Eine weitere Möglichkeit ist die Fütterung von Glucose-1- ${ }^{14} \mathrm{C}$ an Chlorella und die Phosphorolyse der gebildeten Stärke. Da aber dabei eine starke Verschmierung der Radioaktivität vor allem in die Positionen 6, 3 und 4 erfolgt $^{2}$, war auch damit kein befriedigendes Ergebnis zu erwarten.

Diese Schwierigkeiten konnten auf einfache Weise umgangen werden. Wir stellten die positionsmarkierte Saccharose durch Übertragung eines Fructoserestes von Raffinose auf Glucose-1-14C mit einer Transfructosidase her. Das Verfahren hat den Vorteil, daß es von freier Glucose ausgehend ohne Isolierung von Zwischenprodukten in vitro ausgeführt werden kann und mit guter Ausbeute exakt positionsmarkierte Saccharose liefert. Als Transfructosidase benützten wir ein Rohpräparat von „levansucrase“ aus Bacillus subtilis. Zu seiner Gewinnung wurden $2 l$. Nährlösung ( $5 \mathrm{~g}$ Cenovis-BierhefeExtrakt $+10 \mathrm{~g}$ Pepton $+5 \mathrm{~g}$ Saccharose auf $1 l$ Wasser, $\left.p_{H} 6,8\right)$ mit $20 \mathrm{ml}$ einer $13 \mathrm{Stdn}$. alten Kultur beimpft, $13 \mathrm{Stdn}$. bei $30{ }^{\circ} \mathrm{C}$ unter Schütteln bebrütet, die Bakterien abzentrifugiert, 2-mal mit dest. Wasser gewaschen und in der 5-fachen Menge Wasser aufgenommen. Die Suspension wurde bei $4{ }^{\circ} \mathrm{C}$ mit Glasperlen in einer Mickle-Apparatur solange geschüttelt, bis die mikroskopische Kontrolle ergab, daß ungefähr die Hälfte der Bakterien leer war (5 Stdn.). Danach wurde von den Glasperlen abdekantiert und bei $4{ }^{\circ} \mathrm{C}$ und $17000 \mathrm{~g}$ $40 \mathrm{~min}$ zentrifugiert. Die überstehende klare Lösung wird im folgenden als Enzym bezeichnet. Bei einer Lagertemperatur von $-15^{\circ} \mathrm{C}$ hatte ihre Aktivität nach 4. Monaten noch nicht abgenommen.

Die „levansucrase“ kann mehrere Reaktionen katalysieren. Sie überträgt reversibel D-Fructofuranosereste von der Raffinose auf Glucose und bildet so Saccharose $^{3}$. Von der Saccharose wird der Fructoseteil ir-

1 S. Aronoff, Techniques of Radiobiochemistry 1958.

2 O. Kandler u. M. Gibbs, Z. Naturforschg. 14 b, 8 [1959].

3 S. Hestrin, D. S. Feingold u. G. Avigad, J. Amer. chem. Soc. 77, 6710 [1955]. reversibel auf das Polyfructosid Lävan übertragen ${ }^{4}$ und die Glucose zurückgebildet. Bei den anschließend beschriebenen Versuchen tritt weiterhin auch stets eine größere Menge von freier Fructose auf. Man muß daher annehmen, daß das Rohenzym auch hydrolytische Eigenschaften hat. In unseren Ansätzen läuft also eine komplexe Reaktion ab, bei der die Saccharose nur als Intermediärprodukt auftritt. Es mußte daher in Vorversuchen der Zeitpunkt und die Konzentration an Substrat und Enzym ermittelt werden, bei der ein möglichst hoher Anteil von Saccharose vorliegt.

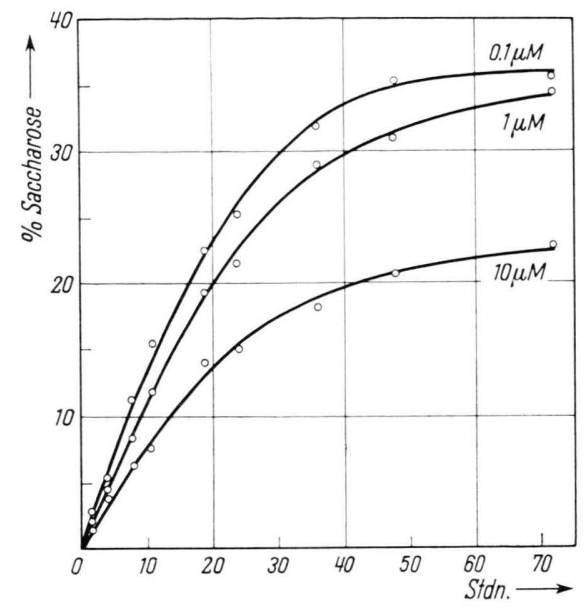

Abb. 1. Einfluß der Glucosekonzentration [ $\mu \mathrm{Mol}]$ auf den Verlauf der Saccharosebildung.

Abb. 1 zeigt den Einfluß von verschiedenen Glucose/ Raffinose-Verhältnissen auf die Saccharosemenge. Die Ansätze für diesen Versuch enthielten in einem Gesamtvolumen von $0,7 \mathrm{ml} 0,2 \mathrm{ml} \mathrm{m} / 50$-Mc-Ilvaine-Puffer $p_{\mathrm{H}}$ 5,5, 0,1 ml Enzym, 0,1 ml Toluol, $10 \mu$ Mole Raffinose und wechselnde Mengen radioaktive Glucose $(0,1,1$, $10 \mu$ Mole). Die Proben wurden bei Zimmertemperatur inkubiert, nach den angegebenen Zeiten jeweils $30 \mu l$ entnommen, auf Chromatographiepapier aufgetragen und eindimensional in Butanol/Propionsäure/Wasser (750 ml n-Butanol $+50 \mathrm{ml} \mathrm{H}_{2} \mathrm{O}$ mit $352 \mathrm{ml}$ Propionsäure $+448 \mathrm{ml} \mathrm{H}_{2} \mathrm{O}$ im Verhältnis $1: 1$ gemischt) getrennt. Bei 0,1 und $1,0 \mu \mathrm{Mol}$ Glucose liegt nach 72 Stdn. ungefähr $35 \%$ der Radioaktivität als Saccharose vor. Ist die Konzentration an Glucose und Raffinose gleich, so ist der Anteil der Saccharose wesentlich geringer.

In Abb. 2 ist der Einfluß der Enzymmenge dargestellt. Die Ansätze enthielten in 1,0 ml Gesamtvolumen $0,2 \mathrm{ml}$ Puffer, 0,1 ml Toluol, $10 \mu$ Mole Raffinose, $1 \mu \mathrm{Mol}$ Glucose $-{ }^{14} \mathrm{C}$ und 0,1 bis $0,6 \mathrm{ml}$ Enzym. Der Anteil der Saccharose an der Radioaktivität erreicht

\footnotetext{
4 S. Hestrin, D. S. Feingold u. G. Avigad, Biochem. J. 64, 340 [1956].
} 
nach einiger Zeit ein Maximum und fällt dann wieder ab. Dabei wird der Maximalwert mit zunehmender Enzymmenge kleiner. Nach dem Auszählen der Radioaktivität wurden die Chromatogramme mit dem Zucker-

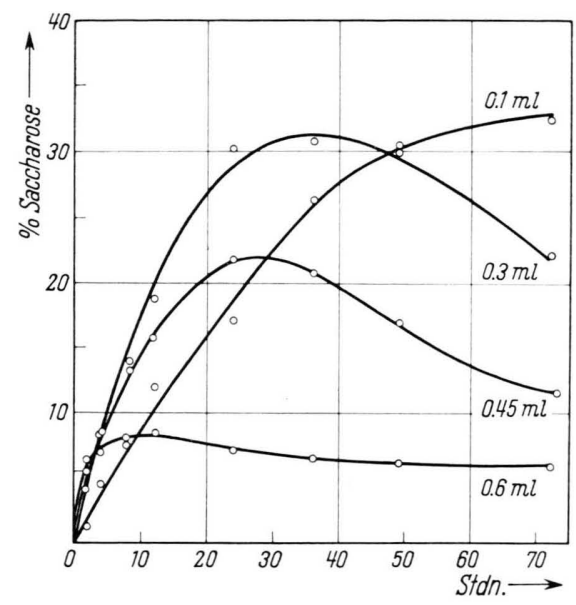

Abb. 2. Einfluß der Enzymmenge [ml] auf den Verlauf der Saccharosebildung.

reagens nach Trevelyan ${ }^{5}$ entwickelt und visuell bewertet. Es ergab sich zum Beispiel für die Versuche mit 0,45 und $0,6 \mathrm{ml}$ Enzym, daß im abfallenden Teil der Kurve die noch vorhandene Raffinosemenge relativ gering war. Möglicherweise ist also eine zu niedrige Raf-

5 W. E. Trevelyan, D. D. Procter u. J. S. Harrison, Nature finosekonzentration für den Abfall verantwortlich. Die freie Fructose tritt mit zunehmender Zeit und steigender Enzymmenge in immer stärkerem Maße auf. Es legt dies den Gedanken nahe, daß die Transfructosylierung und die Hydrolyse vielleicht Fähigkeiten eines einzigen Enzyms in der „levansucrase“ sind. Ähnliches konnte für eine Reihe von Glycosidasen (Invertase, Lactase, Cellobiase etc.) bewiesen werden ${ }^{6}$.

Auf Grund dieser Ergebnisse wurde für die Synthese der positionsmarkierten Saccharose das Verhältnis von Glucose zu Raffinose wie 1: 10 gewählt. Die Enzymkonzentration entsprach ungefähr dem Versuch mit $0,3 \mathrm{ml}$ in Abb. 2. Der Ansatz enthielt in $6 \mathrm{ml} \mathrm{Gesamt-}$ volumen $2 \mathrm{ml}$ Enzym, $2 \mathrm{ml}$ Puffer, 0,1 ml Toluol, $138 \mu$ Mole Raffinose und $13,8 \mu$ Mole Glucose-1-14C (spez. Aktivität $7,2 \mu \mathrm{C} / \mu \mathrm{Mol}$ ). Nach $36 \mathrm{Stdn}$. Inkubation bei Zimmertemperatur wurde die Probe im Luftstrom eingeengt, in 35 Streifen von $6 \mathrm{~cm}$ Länge auf Whatman Nr. 1 aufgetragen und in Butanol/Propionsäure $/ \mathrm{H}_{2} \mathrm{O}$ eindimensional chromatographiert. In diesem Lösungsmittel werden alle an der Reaktion beteiligten Zucker einwandfrei getrennt. Die nicht umgesetzte Glucose wird daher in unveränderter spez. Aktivität und Reinheit zurückgewonnen. Die erhaltene Saccharose (ungefähr $37 \mu \mathrm{C}$ ) wurde vom Papier eluiert und nochmals chromatographiert. Kleine Anteile davon wurden in 6 Lösungsmittelsystemen mit authentischem Material cochromatographiert. Dabei deckten sich Radioaktivität und Substanzfleck stets vollkommen. Nach der Behandlung mit melibiasefreier Invertase ergab sich, daß die Radioaktivität ausschließlich in der Glucose enthalten ist.

[London] 166, 444 [1950].

6 J. Edelman, Advances in Enzymol. 17, 189 [1956].

\section{Stoff wechseluntersuchungen an Pflanzen mit ${ }^{14} \mathrm{C}$}

\section{Von W. Häussermann und W. Rentschler}

Institut für Physik und Meteorologie der Landwirtschaftlichen Hochschule Hohenheim

(Z. Naturforschg. 17 b, 699-701 [1962]; eingegangen am 16. März 1962)

Zur Erprobung einer Meßanordnung zur Untersuchung der Abgabe von Kohlenstoffverbindungen bei Pflanzen mit Hilfe ${ }^{14} \mathrm{C}$-markierten Kohlenstoffs wurde die Ausscheidungsgeschwindigkeit von frisch aufgenommenem ${ }^{14} \mathrm{CO}_{2}$ bei einem etwa $50 \mathrm{~cm}$ hohen im Gewächshaus angezogenen jungen Apfelbaum (Pirus malus) untersucht.

Zum Einbau des markierten Kohlenstoffes ${ }^{1,2}$ wurden die oberirdischen Teile der Versuchspflanze in einen Polyäthylen-Beutel von $20 l$ Inhalt eingeschlossen. In diesen Beutel wurde das aus einer $\mathrm{Na}_{2}{ }^{14} \mathrm{CO}_{3}$. Lösung entwickelte markierte $\mathrm{CO}_{2}$ eingebracht. Die ins-

1 D. SaUerbeck, Atompraxis 6, 221 [1960].

2 H. W. Scharpenseel, Landwirtsch. Forsch. 14, 42 [1961]. gesamt zugeführte ${ }^{14} \mathrm{C}$-Aktivität betrug $0,5 \mathrm{mc}$. Dabei waren $60 \%$ der eingebrachten $\mathrm{CO}_{2}$-Moleküle markiert. In diesem Beutel blieb die Pflanze eine halbe Stunde. Während dieser Zeit wurde sie mit 20000 Lux (zwei Osram Concentra-Lampen $150 \mathrm{~W}$ und Wasserfilter $5 \mathrm{~cm}$ ) beleuchtet. Daraufhin wurde die Pflanze eine halbe Stde. im Freien ohne Beutel aufgestellt, damit eventuell nur oberflächlich adsorbiertes ${ }^{14} \mathrm{CO}_{2}$ abgegeben werden konnte. Dann wurde die ganze Pflanze mit einem neuen Polyäthylen-Beutel von $20 l$ Inhalt umkleidet, durch den während des weiteren Versuches Außenluft mit konstanter Strömungsgeschwindigkeit $(12 l / h)$ gesaugt wurde. Der Pflanze wurden dabei $2 \mathrm{mg} \mathrm{CO} \mathrm{CO}_{2}$ pro Stde. und $\mathrm{dm}^{2}$ Blattfläche zugeführt. Die Luftfeuchtigkeit in der Kammer betrug ständig 100 Prozent. Die ${ }^{14} \mathrm{C}$-Aktivität der abgesaugten Luft wurde nach deren Trocknung mit konzentrierter Schwefelsäure in einer Ionisationskammer von $200 \mathrm{~cm}^{3}$ Inhalt bestimmt. Zur Messung des Ionisationsstromes diente ein Schwingkondensator-Verstärker, dessen Ausgangsspannung mit einem elektronischen Kompensographen registriert wurde. Sowohl die Blatt- und Umgebungstempe- 